\title{
El papel del profesor en la motivación y la salud mental de los estudiantes de educación física
}

\author{
Jorge Zamarripa, ${ }^{1}$ Isabel Castillo, ${ }^{2}$ Inés Tomás, ${ }^{2}$ José Tristán, ${ }^{1}$ Octavio Álvarez ${ }^{2}$
}

Artículo original

\begin{abstract}
Introduction

Self-Determination Theory (SDT) suggests that individuals with autonomous regulations hold greater levels of optimal functioning than those with controlling regulations. In addition, SDT underlines the importance of social aspects within motivational processes. In the educational setting, teachers' interpersonal style while giving instructions represents one of the most prominent social factors for triggering students' motivation. Therefore, contexts that foster autonomous motivation are considered the most suitable for promoting pupils' optimal growth and mental health.
\end{abstract}

\section{Objective}

To examine if students' perception of Physical Education Teachers' support of their needs of autonomy, competence and relatedness could help encouraging their well-being and reducing their ill-being, through the mediating role of motivation.

\section{Method}

Participants were 734 secondary school students between 11-17 years old. We used a cross-sectional design. Statistical analyses were carried out using structural equation modeling.

\section{Results}

The degree to which the students perceived that the teacher-created context supported their autonomy, competence and relatedness predicted positively their autonomous motivation and negatively their no motivation, which in turn predicted positively well-being and negatively ill-being.

\section{Discussion and conclusion}

Our results highlight the importance of autonomous motivation as the most powerful mediation mechanism for improving well-being and avoiding ill-being in physical activity classes. This research confirms and extends the finding of the self-determination theory within the Mexican population.

Key words: Faculty, physical education and training, motivation, mental health, students, Mexico.

\section{RESUMEN}

\section{Introducción}

La teoría de la autodeterminación sugiere que los sujetos con regulaciones autónomas mostrarán mayores niveles de funcionamiento óptimo que aquellos con regulaciones controladoras. Asimismo, la teoría ha defendido la importancia que tienen los factores sociales en los procesos motivacionales. En el contexto educativo, uno de los factores sociales que tiene un papel importante en la motivación de los estudiantes es el estilo interpersonal que utiliza el profesor cuando brinda instrucciones; por tanto, aquellos ambientes que fomenten la motivación autónoma serán importantes para el desarrollo óptimo y la salud mental de los estudiantes.

\section{Objetivo}

Examinar si el grado en que los estudiantes perciben que el profesor de educación física apoya sus necesidades de autonomía, competencia y relaciones ayudará a fomentar su bienestar y reducir su malestar, a través de la motivación como mecanismo mediador.

\section{Método}

Participaron 734 estudiantes de secundaria entre 11 y 17 años usando un diseño de estudio transversal. Se utilizó la técnica estadística de ecuaciones estructurales.

\section{Resultados}

Los alumnos que perciben que su profesor apoya su autonomía, su competencia y sus relaciones presentan mayor motivación autónoma y menor no motivación; lo que les genera mayor bienestar y menor malestar.

\section{Discusión y conclusión}

Se destaca la importancia de la motivación autónoma como el mecanismo mediador más potente para generar bienestar y evitar malestar en las clases de actividad física. Este estudio extiende y generaliza los hallazgos de la teoría de la autodeterminación a la población mexicana.

Palabras clave: Profesores, educación física, motivación, salud mental, estudiantes, México.

Facultad de Organización Deportiva, Universidad Autónoma de Nuevo León, México.

2 Facultad de Psicología, Universitat de València, España.

Correspondencia: Dr. Jorge Zamarripa. Facultad de Organización Deportiva, Universidad Autónoma de Nuevo León, Cd. Universitaria, s/n, 66451, San Nicolás de los Garza, NL, México. Tel. + 52 (81) 1340 - 4450 y 51 (Ext. 7634). Fax: 7640. E-mail: jorge.zamarriparv@uanl.edu.mx

Recibido primera versión: 3 de septiembre de 2015. Segunda versión: 4 de febrero de 2016. Aceptado: 7 de julio de 2016. 


\section{INTRODUCCIÓN}

La educación física ha sido reconocida por muchos especialistas como uno de los principales medios para promover la salud y el bienestar de las personas, ya que se ofrece en un contexto que permite llegar a un gran número de niños, adolescentes y jóvenes. ${ }^{1,2}$ Para lograr esto, es necesario que profesores, directores de centros educativos e instancias de gobierno, comprendan que la motivación es una herramienta fundamental que debe ser utilizada en las sesiones de clase. ${ }^{3}$

La Teoría de la Autodeterminación (Self-Determination Theory; SDT por sus siglas en ingles $)^{4,5}$ postula que la conducta puede estar motivada de modo intrínseco, extrínseco o puede no estar motivada. Estos tres tipos de motivación varían en su grado de autodeterminación o autonomía, pudiendo situarse en un continuo de la autodeterminación donde la conducta oscilaría desde altos niveles de autonomía (motivación intrínseca), pasando por niveles medios (motivación extrínseca), hasta bajos niveles (no motivación). Un alumno intrínsecamente motivado se implicará en una actividad por la satisfacción y el placer que le produce la propia actividad. ${ }^{4}$ Aquellos alumnos que se implican en las actividades por las consecuencias derivadas de su realización estarán extrínsecamente motivados, y su conducta estará guiada por distintas regulaciones extrínsecas según se avanza en el continuo de la autodeterminación. La regulación menos autónoma es la externa, y se refiere a la realización de un comportamiento para obtener una recompensa o evitar un castigo. La regulación introyectada es cuando el comportamiento se realiza para evitar la culpa o la vergüenza. La regulación extrínseca más autónoma es la identificada y se produce cuando el alumno acepta y valora los beneficios asociados con la actividad. Desde la SDT se indica que estas regulaciones se agrupan formando la motivación autónoma (intrínseca e identificada) versus la motivación controlada (introyectada y externa). ${ }^{6}$ Por último, aquellos alumnos sin motivación serán los que no tienen intención de realizar las actividades, no estando ni intrínseca ni extrínsecamente motivados. Las personas no motivadas, son incapaces de percibir una contingencia entre su comportamiento y los resultados subsecuentes de este comportamiento. ${ }^{4}$

La SDT sugiere que los sujetos con regulaciones más autónomas mostrarán mayores niveles de funcionamiento óptimo que aquellos con regulaciones más controladoras. ${ }^{6}$ Los resultados de los estudios que han examinado las consecuencias de las regulaciones motivacionales sobre diferentes indicadores de bienestar apoyan la postura de que las regulaciones más autodeterminadas se encuentran asociadas con el esfuerzo, ${ }^{7}$ la concentración, los afectos positivos y la predisposición a realizar tareas desafiantes, ${ }^{3}$ la persistencia de la práctica fuera de la escuela, ${ }^{8}$ el aprendizaje durante la clase ${ }^{9}$ y el disfrute. ${ }^{10}$ Por otro lado, las regulaciones más controladoras y la no motivación se encuentran asociadas con indicadores de malestar como el aburrimiento, el estado depresivo y la infelicidad., 3,11,12 Por tanto, se puede considerar que aquellos ambientes en los que se fomente la motivación autónoma serán importantes para el desarrollo óptimo y para la salud mental. Dado que los intereses, habilidades y esfuerzo que emplean los alumnos en la clase de educación física difieren unos de otros, resulta interesante comprender el papel del profesor para crear climas motivacionales adecuados y su efecto sobre el bienestar y malestar de los estudiantes durante la sesión.

Desde la SDT se ha defendido la importancia que tienen los factores sociales en los procesos motivacionales. En el contexto educativo, uno de los factores sociales que tiene un papel importante en la motivación de los estudiantes es el estilo interpersonal que utiliza el profesor cuando brinda instrucciones a sus estudiantes. Así, un profesor que en su estilo de enseñanza apoye la autonomía (i.e. que considere la perspectiva del estudiante proporcionando explicaciones sobre el porqué de las actividades a realizar), la competencia (i.e. ajustando los objetivos según las habilidades de los estudiantes) y que fomente las buenas relaciones entre los alumnos (i.e. mostrando interés y apoyo emocional al estudiante) potenciará una motivación autodeterminada, promoviendo el bienestar, el buen funcionamiento de los estudiantes y evitando el malestar de los mismos.,33,14

A nivel empírico, la mayoría de los trabajos realizados en el contexto educativo se han centrado principalmente en examinar el apoyo del profesor a la autonomía del alumno, ${ }^{15-18}$ y aunque algunos trabajos ya han evidenciado la necesidad de incluir el apoyo a la competencia y las relaciones para estudiar los antecedentes de la motivación de los estudiantes y sus consecuencias, ${ }^{3,8,19-22}$ pocos son los que han examinado la relación entre los tres tipos de apoyo de forma conjunta y la motivación en la educación física. ${ }^{3,8}$

Con la finalidad de cubrir estas deficiencias, en este estudio se considerará no solamente el apoyo a la autonomía (como se ha hecho en la mayoría de los estudios previos), sino que también se incluirá el apoyo a la competencia y a las relaciones.

El objetivo del presente estudio ha sido, en primer lugar, poner a prueba un modelo donde se hipotetiza que el grado en el que los estudiantes perciben que su profesor apoya su autonomía, su competencia y sus relaciones, afectará al bienestar y malestar de los estudiantes, con la motivación como mecanismo mediador. Concretamente, el apoyo del profesor estará positivamente relacionado con la motivación autónoma y negativamente con la motivación controlada y con la no motivación. Por su parte, la motivación autónoma estará positivamente relacionada con los afectos positivos (como indicador de bienestar) y negativamente con los afectos negativos (como indicador de malestar); y al contrario, la motivación controlada y la no motivación estarán relacionados de manera positiva con los afectos negativos y de forma negativa con los afectos positivos. 


\section{MÉTODO \\ Diseño del estudio y descripción de la muestra}

Se realizó un estudio transversal descriptivo en el que participaron 734 estudiantes de secundaria (393 varones y 341 mujeres) del municipio de San Nicolás de los Garza en Nuevo León, México, con una edad media de 13.7 años $(D E=1.09$; rango $=11-17$ años $)$. La distribución por cursos fue la siguiente: $33.8 \%$ cursaban el primer grado, $32.4 \%$ cursaban el segundo grado y $33.8 \%$ cursaban el tercer grado. La mayoría de ellos pertenecía a escuelas secundarias públicas $(84.5 \%)$ y solamente un pequeño porcentaje asistía a institutos privados (15.5\%).

\section{Procedimiento}

Para seleccionar a los participantes se utilizó un muestreo por conveniencia considerando tanto escuelas públicas como privadas, y asegurando una representatividad proporcional de los distintos grados. Se pidió autorización a los centros educativos mediante una carta donde se explicaban los objetivos y el procedimiento de investigación. La recopilación de información se realizó de forma auto-administrada y anónima en una aplicación colectiva en clase. Se solicitó consentimiento informado de los progenitores o tutores de los participantes. La recopilación de los datos se realizó durante los meses de abril y mayo del año 2013. Todos los sujetos invitados a participar cumplimentaron la información solicitada.

\section{Instrumentos}

La percepción del apoyo del profesor se evaluó utilizando la versión mexicana ${ }^{23}$ del Cuestionario de Apoyo a las Necesidades Psicológicas Básicas adaptada a la Educación Física (CANPB), compuesto por 24 ítems de los cuales 15 miden el apoyo a la autonomía, cuatro el apoyo a la competencia y cinco el apoyo a las relaciones (p.e., "el (la) maestro(a) pone atención a cómo nos gustaría hacer las cosas"). En el encabezado se presenta la frase "En esta clase de educación física..." Las respuestas se recogen en una escala Likert de siete puntos ( 1 = "muy en desacuerdo"; 7 = "muy de acuerdo"). Investigaciones previas han informado una adecuada fiabilidad de este instrumento, confirmando una estructura unifactorial ${ }^{3,8,23} \mathrm{o}$ trifactorial. ${ }^{21,23}$

La motivación se evaluó con la escala de Percepción del Locus de Causalidad. ${ }^{24}$ Está compuesta por 20 ítems, cuatro para cada subescala: motivación intrínseca, regulación identificada, regulación introyectada, regulación externa, y no motivación. La escala está precedida de la frase: "Participo en esta clase de educación física...". Un ejemplo de ítem sería "porque es divertida". Los estudiantes respondieron en una escala Likert de siete puntos ( 1 = "muy en desacuerdo"; 7 = "muy de acuerdo"). Para la variable de motivación autónoma se combinaron los ítems de motivación intrínseca y regulación identificada; para la motivación controlada, se combinaron los ítems de regulación introyectada y externa. Estudios previos han confirmado la fiabilidad de esta escala en la educación física., ${ }^{3,21}$

Se utilizó el inventario de afectos positivos y negativos, ${ }^{25}$ el cual ha sido utilizada previamente por otros estu$\operatorname{dios}^{26}$ como indicadores de bienestar y malestar. Esta escala consta de nueve ítems: cinco para afectos positivos y cuatro para afectos negativos, que indican el grado en el que los estudiantes experimentan cada afecto sobre una escala de cinco puntos ( 1 = "nunca"; 5 = "siempre"). Los ítems estaban precedidos por la frase: "En la clase de educación física me siento...". Un ejemplo de ítem sería: "feliz". Trabajos previos han confirmado la fiabilidad de estas escalas en la educación física. ${ }^{3}$

\section{Análisis estadísticos}

El número de datos faltantes fue tan escaso (.003\%) que no se consideró necesario realizar imputación de los datos.

En primer lugar se realizaron análisis factoriales confirmatorios (AFC) de cada instrumento por separado. Para el CANPB se pusieron a prueba dos modelos, de uno y tres factores respectivamente; para las motivaciones se puso a prueba un modelo trifactorial; y para los afectos un modelo bifactorial.

El ajuste de los modelos se examinó utilizando diversos índices: RMSEA, NNFI y CFI. Valores de RMSEA igual o por debajo de .08 y de .10 indican un ajuste óptimo y satisfactorio respectivamente. Valores de NNFI y CFI por encima de .95 indican un ajuste satisfactorio. En este sentido, diferentes estudios, ${ }^{27,28}$ sugieren no ignorar los efectos de la complejidad del modelo cuando se evalúan los índices de ajuste. Los puntos de corte convencionales pueden resultar muy restrictivos cuando se aplican a modelos complejos. Para determinar cuál de los dos modelos puestos a prueba para el CANPB ajustaba mejor, se analizaron las diferencias entre ambos. Diferencias entre los valores NNFI y los valores CFI inferiores a .01, y diferencias entre los valores RMSEA inferiores a .015, indican diferencias irrelevantes, mostrando apoyo para utilizar el modelo más parsimonioso. ${ }^{27,29}$

En segundo lugar, se realizaron análisis descriptivos y de fiabilidad de las escalas utilizando el alfa de Cronbach. En tercer lugar, se puso a prueba el modelo de ecuaciones estructurales hipotetizado (figura 1), utilizando variables latentes. Las matrices input fueron las de correlaciones policóricas y de covarianzas asintóticas, y dado que el tamaño muestral no permitía utilizar el método de Mínimos Cuadrados Ponderados, el método de estimación utilizado fue el de Máxima Verosimilitud, utilizando el programa LISREL $8.80 .^{30}$ Primero se analizó el modelo total de medida, 


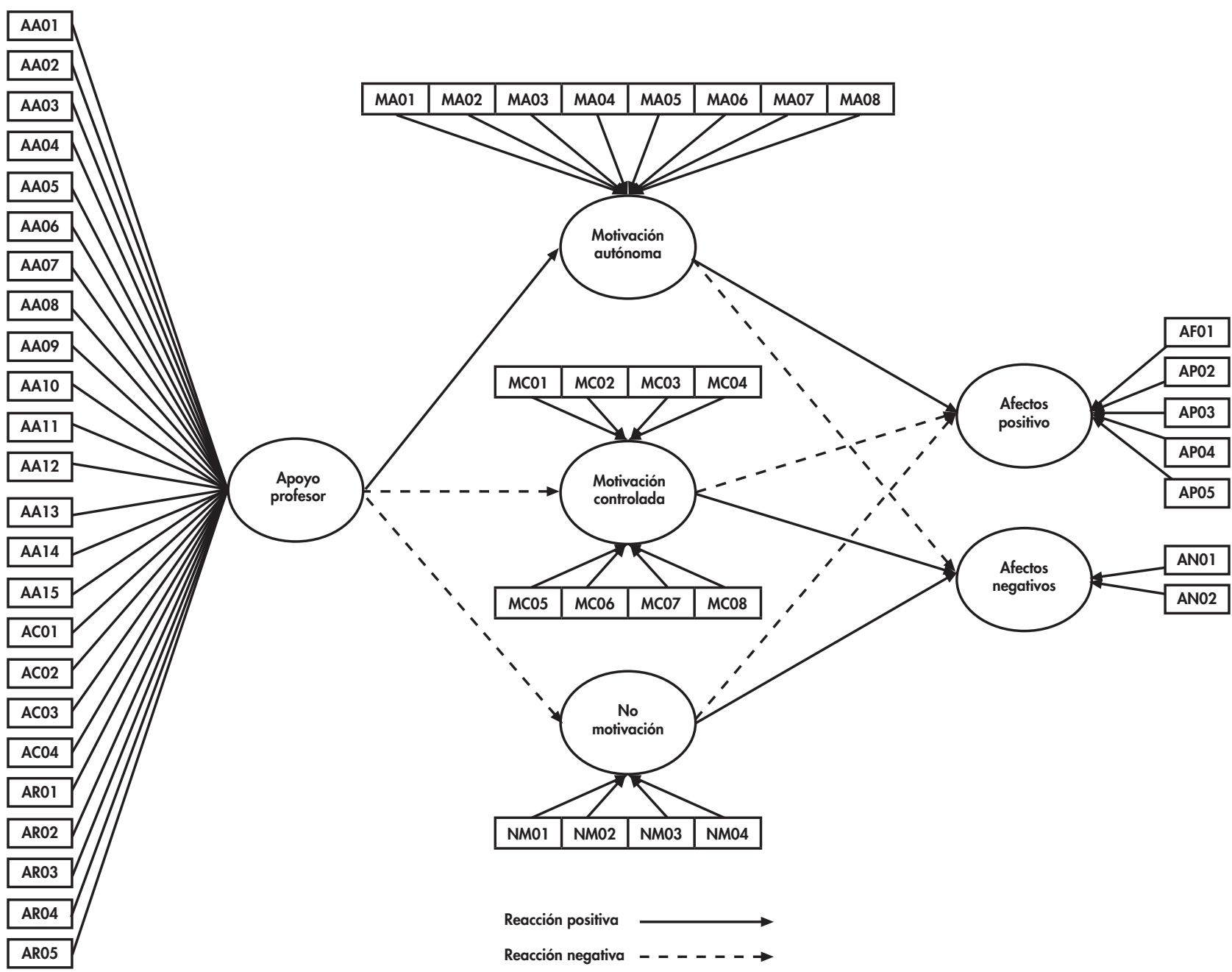

Figura 1. Modelo estructural hipotetizado del apoyo del profesor, la motivación y los afectos. Estudio con estudiantes en San Nicolás de los Garza, Nuevo León, México, 2012.

que resulta equivalente a un modelo factorial confirmatorio, poniendo además a prueba la validez divergente de las variables latentes, y, posteriormente, se evaluó el modelo estructural. El ajuste se examinó con los mismos índices utilizados para el AFC.

En cuarto lugar, se evaluaron los efectos de mediación utilizando el procedimiento del producto de coeficientes. ${ }^{31,32}$ Adicionalmente, en los casos en los que se confirmó el efecto de mediación, se evaluó si era total o parcial. Finalmente, se llevaron a cabo pruebas de contraste para evaluar diferencias entre los efectos de mediación encontrados. ${ }^{33}$

\section{RESULTADOS}

\section{Análisis factoriales confirmatorios}

Los índices de bondad de ajuste de los modelos unifactorial y trifactorial del CANPB fueron adecuados [unifactorial: $\chi^{2}(252)=682.89, \mathrm{NNFI}=.963, \mathrm{CFI}=.966$ y RMSEA $=.048$; trifactorial: $\chi^{2}(249)=631.04, \mathrm{NNFI}=.967, \mathrm{CFI}=.970$ y RMSEA $=.046]$. Se comprobó que las diferencias entre los índices de ajuste fueron irrelevantes $(\Delta \mathrm{NNFI}=.004, \Delta \mathrm{CFI}=.004$ y $\Delta$ RMSEA = .002), por lo que se decidió utilizar el modelo más parsimonioso (unifactorial). Además, la alta correlación entre los factores del modelo trifactorial (correlaciones entre $.969 \mathrm{y}$ .997) sugieren singularidad, por lo que el modelo unifactorial resultó ser el más adecuado. Respecto a la escala de motivación, los índices de ajuste del modelo trifactorial fueron satisfactorios $\left[\chi^{2}(167)=1144.07, \mathrm{NNFI}=.921, \mathrm{CFI}=.931\right.$ y RMSEA $=.089$ ]. Por último, los índices de ajuste del modelo bifactorial de la escala de afectos positivos y negativos fueron satisfacto$\operatorname{rios}\left[\chi^{2}(26)=116.12, \mathrm{NNFI}=.923, \mathrm{CFI}=.939\right.$ y $\left.\mathrm{RMSEA}=.068\right]$.

\section{Descriptivos y fiabilidad}

Los descriptivos y fiabilidad de las medidas figuran en el cuadro 1. Las medidas mostraron una alta fiabilidad, a ex- 
Cuadro 1. Estadísticos descriptivos, consistencia interna y correlaciones entre las variables. Estudio con estudiantes en San Nicolás de los Garza, Nuevo León, México, 2012

\begin{tabular}{lccccccccc}
\hline & Rango & $M$ & $D E$ & $\alpha$ & 1 & 2 & 3 & 4 & 5 \\
\hline 1. Apoyo del profesor & $1-7$ & 5.32 & $(1.20)$ & 0.95 & - & & & & \\
2. Mot. autónoma & $1-7$ & 5.78 & $(1.27)$ & 0.93 & $0.59^{* *}$ & - & & & \\
3. Mot. controlada & $1-7$ & 4.16 & $(1.38)$ & 0.82 & $0.21^{* *}$ & $0.30^{* *}$ & - & \\
4. No motivación & $1-7$ & 2.52 & $(1.60)$ & 0.84 & $-0.16^{* *}$ & $-0.23^{* *}$ & $0.33^{* *}$ & - & \\
5. Afectos positivos & $1-5$ & 3.84 & $(0.82)$ & 0.81 & $0.52^{* *}$ & $0.65^{* *}$ & $0.17^{* *}$ & $-0.19^{* *}$ & - \\
6. Afectos negativos & $1-5$ & 1.69 & $(0.73)$ & - & $-0.26^{* *}$ & $-0.36^{* *}$ & -0.04 & $0.21^{* *}$ & $-0.41^{* *}$ \\
\hline
\end{tabular}

Nota: Mot.: Motivación; M: Media; DE: Desviación estándar; $\alpha$ : alfa de Cronbach.

${ }^{* *} p<0.01$. Para los Afectos Negativos el valor de $r=0.41$.

cepción de la de afectos negativos que fue de .55. Un análisis de la consistencia sugería eliminar los ítems "culpable" y "nervioso" por presentar correlaciones ítem-total por debajo de .30, por lo que se utilizaron los ítems "enojado" e "infeliz" como indicadores de afectos negativos.

\section{Modelo de medida y estructural}

El modelo de medida presentó un ajuste satisfactorio $\left[\chi^{2}(1160)=6610.34 ;\right.$ CFI $=0.958 ; \mathrm{NNFI}=0.955 ;$ RMSEA $=$ $0.080]$, confirmando la validez divergente de las variables latentes. El modelo estructural también mostró índices de ajuste aceptables $\left[\chi^{2}(1162)=6691.02 ; \mathrm{CFI}=.957 ; \mathrm{NNFI}=\right.$ .955 ; RMSEA = .081]. El apoyo del profesor se asoció positivamente con la motivación autónoma y negativamente con la no motivación. La motivación autónoma se asoció positivamente con los afectos positivos y negativamente con los afectos negativos. Mientras que la no motivación se asoció positivamente con los afectos negativos (figura 2) (no se presentan las estimaciones de las saturaciones factoriales ni los términos de error, quedando éstos a disposición de quien

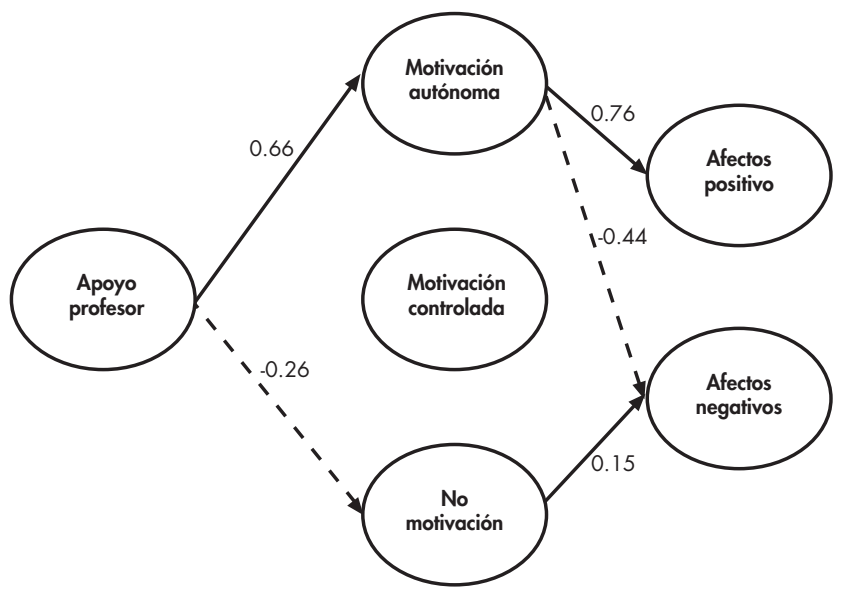

Figura 2. Solución estandarizada del modelo estructural hipotetizado del apoyo del profesor, la motivación y los afectos. Estudio con estudiantes en San Nicolás de los Garza, Nuevo León, México, 2012.

Nota. Los coeficientes son estandarizados y significativos $(p<.05)$. lo requiera al primer autor). El modelo explicó el 42\% de la varianza de la motivación autónoma, el 1\% de la motivación controlada, el $7 \%$ de la no motivación, el 58\% de los afectos positivos y el $27 \%$ de la varianza de los afectos negativos.

\section{Efectos de mediación y pruebas de contraste}

Se identificaron tres efectos de mediación. Por un lado, la motivación autónoma resultó ser un mediador significativo en la relación entre el apoyo del profesor y el indicador de bienestar $\left(\alpha_{1} \beta_{1}=.42 ;\right.$ IC 95\% = [.34, .50]). Además, se confirmó que dicho efecto de mediación era parcial $(\tau=.21, p<.01)$. Por otro lado, en la relación entre el apoyo del profesor y el indicador de malestar se confirmó el efecto mediador tanto de la motivación autónoma $\left(\alpha_{1} \beta_{2}=-.26\right.$; IC 95\% = [-.37, -.15]), como de la no motivación $\left(\alpha_{2} \beta_{3}=-.04\right.$; IC 95\% = [-.08, -.01]), tratándose en ambos casos de una mediación total $(\tau=-.06, p>.05)$.

El contraste entre el efecto de mediación de la motivación autónoma y de la no motivación, en la relación entre el apoyo del profesor y el indicador de malestar resultó estadísticamente significativo ( $\psi=.22$; IC 95\% = [.09, .35]).

Finalmente, la motivación autónoma resultó ser mediador tanto de la relación entre el apoyo del profesor y el indicador de bienestar, como del apoyo del profesor y el indicador de malestar. Los resultados indicaron que el contraste entre ambos efectos de mediación no resultó estadísticamente significativo $(\psi=.16$; IC 95\% $=[-.01, .31])$.

\section{DISCUSIÓN Y CONCLUSIÓN}

El presente estudio pone a prueba un modelo en el que se hipotetizó que el grado en que los estudiantes perciben que el profesor apoya su autonomía, su competencia y sus relaciones estaría relacionado con los diferentes tipos de motivación, y éstos a su vez se relacionarían con el bienestar/ malestar de los estudiantes.

Los resultados muestran que la percepción de los estudiantes sobre el apoyo que el profesor brinda a la autonomía, la competencia y las relaciones se asociaron de forma positiva con la motivación autónoma, y de forma negativa con la no motivación. Contrario a nuestra hipótesis, el apoyo 
a las necesidades psicológicas básicas no se asoció de forma significativa con la motivación controlada, aunque sí se encontró una asociación positiva entre ambas variables. Como indican estudios previos, ${ }^{3}$ puede ser que los antecedentes de las regulaciones menos autodeterminadas sean distintos en el contexto de la educación física en relación con otros contextos sociales, y que el apoyo que brinda el profesor no reduzca las presiones externas a las que los alumnos se ven sometidos para realizar sus actividades en el ámbito académico (i.e., culpa, obligación, castigo, etc.).

Respecto a la asociación entre el apoyo del profesor y las regulaciones más autónomas de la motivación en las clases de educación física, nuestros resultados también concuerdan con estudios previos ${ }^{8,17}$ y confirman que si el profesor adopta un estilo interpersonal que apoye la autonomía del alumno, si le proporciona óptimos desafíos y buenas relaciones, se incrementan sus niveles de autodeterminación para participar en la clase de educación física, y disminuye su no motivación.

En cuanto a la influencia de los diferentes tipos de motivación sobre los afectos positivos y negativos, los resultados de este estudio revelaron que los afectos positivos se asociaron positivamente con la motivación autónoma, mientras que los afectos negativos se asociaron negativamente con dicha motivación y positivamente con la no motivación. Estos resultados van en la línea de estudios realizados en el contexto de la educación física ${ }^{9,10}$ y muestran que los estudiantes que tienen una motivación autónoma durante la clase de educación física experimentan más sensaciones positivas de dicha participación (como placer y satisfacción derivada de hacerlo) y menos sensaciones negativas derivadas de dicha participación (como sentirse enojados o infelices). Por el contrario, aquellos que se sientan desmotivados para participar en la clase, se sienten enojados o infelices durante ella. Contrariamente a lo hipotetizado, la motivación controlada no se asoció de forma significativa con los afectos de los estudiantes, aunque sí se apreció una relación positiva y significativa con los afectos positivos. Este resultado es consistente con trabajos previos, ${ }^{3,7}$ lo que hace necesario realizar más estudios que aporten luz a la hipotetizada relación entre la motivación controlada y sus consecuencias desadaptativas.

Nuestros hallazgos remarcan la importancia del apoyo que el profesor debería brindar a la autonomía y competencia de los estudiantes y las relaciones entre los mismos durante la sesión de educación física, ya que este tipo de ambientes sociales fomentan la motivación más auto-determinada, con lo que se incrementan las experiencias positivas en la clase y sensaciones de bienestar, disminuyendo al mismo tiempo las experiencias negativas y las sensaciones de malestar. Asimismo, el contraste del papel mediador de la motivación autónoma y de la no motivación entre el apoyo del profesor y los afectos de los estudiantes ha puesto de manifiesto que el papel que juega la motivación autónoma es más relevante que la no motivación en su relación con los afectos negativos, indicando que cuando los alumnos perciben que sus profesores les apoyan, se ve incrementada su motivación autónoma, lo que les lleva a que aumente su bienestar y disminuyan sus experiencias de malestar. No obstante, aunque en menor medida, la percepción de apoyo del profesor por parte de los estudiantes posee un efecto significativo sobre la no motivación haciendo que ésta disminuya, lo que conlleva una disminución del malestar. Además, se ha confirmado que el papel mediador de la motivación autónoma es igualmente relevante para aumentar los afectos positivos como para disminuir los afectos negativos.

Este trabajo tiene implicaciones teóricas y prácticas. Entre las implicaciones teóricas podemos destacar la importancia de considerar el apoyo de las necesidades psicológicas básicas, incluyendo tanto el apoyo a la autonomía, como de competencia y de relación. Además, se ha puesto de manifiesto la relevancia de fomentar la motivación autónoma tanto para incrementar, a través de ella, las experiencias de bienestar, como para reducir las experiencias de malestar. Finalmente, los resultados de este estudio permiten generalizar los postulados de la SDT a otras poblaciones y contextos culturales, como es el mexicano.

Así mismo, este estudio presenta algunas limitaciones relativas a las características específicas de la muestra de estudio ya que se trata de estudiantes de secundaria, así como el que se trate de un estudio transversal. No obstante, estas limitaciones indican posibles direcciones para futuras investigaciones, ya que sería interesante ampliar el estudio con alumnos de primaria y realizar una recogida longitudinal de datos para fortalecer las conclusiones sobre las relaciones de predicción de las variables incluidas en el estudio.

Por otra parte, las implicaciones prácticas del presente trabajo se centran sobre el diseño de programas de formación y capacitación que guíen a los educadores físicos a planificar, estructurar y desarrollar clases que generen estilos interpersonales que fomenten los tres tipos de apoyo, con la finalidad de que los estudiantes consigan experiencias positivas que les lleven a interesarse por la educación física dentro de la escuela y a adoptar estilos de vida saludables fuera de ella.

\section{Financiamiento}

Programa de Mejoramiento del Profesorado (PROMEP/103.5/12/ 3585). Consejo Nacional de Ciencia y Tecnología, programa de estancias posdoctorales al extranjero para la consolidación de grupos de investigación (203998).

\section{Conflicto de intereses}

Los autores declaran no tener ningún conflicto de intereses.

\section{Agradecimientos}

Se agradece a la profesora doctora Isabel Balaguer Solá y a la Unidad de Investigación de Psicología del Deporte de la Universidad de Valencia, el apoyo y las facilidades prestadas para desarrollar el presente trabajo. 


\section{REFERENCIAS}

1. Sallis J, Owen N. Physical activity \& behavioral medicine. Tercera edición. California: SAGE; 1999; 210.

2. Sallis J. Influences on physical activity of children, adolescents, and adults. PCPFS Research Digest 1999;1(7):1-4.

3. Standage M, Duda JL, Ntoumanis N. A test of self-determination theory in school physical education. Br J Educ Psychol 2005;75(3):411433.

4. Deci E, Ryan R. Intrinsic motivation and self-determintion in human behavior. New York: Plenum Press; 1985; p.371.

5. Deci E, Ryan R. Handbook of self-determination research. New York: The University Rochester Press; 2002; p.449.

6. Deci E, Ryan R. The "what" and "why" of goal pursuits: Human needs and the self-determination of behaviour. Psychol Inq 2000;11(4):227268.

7. Ntoumanis N. A self-determination approach to the understanding of motivation in physical education. Br J Educ Psychol 2001;71(2):225-242.

8. Zhang T, Solmon MA, Kosma M, Carson RL et al. Need support, need satisfaction, intrinsic motivation, and physical activity participation among middle school students. J Teach Phys Educ 2011;30(1):51-68.

9. Grasten A, Jaakkola T, Liukkonen J, Watt A et al. Prediction of enjoyment in school physical education. J Sports Sci Med 2012;11:260-269.

10. Bryan CL, Solmon MA. Student motivation in physical education and engagement in physical activity. J Sport Behav 2012;35:267-285.

11. Ryan R, Deci E. Intrinsic and extrinsic motivations: Classic definitions and new directions. Contemp Educ Psychol 2000;25(1):54-67.

12. Vallerand $\mathbf{R}$, Bissonnette $R$. Intrinsic, extrinsic, and amotivational styles as predictors of behavior: A prospective study. J Pers 1992;60(3):599-620.

13. Ntoumanis N, Standage M. Motivation in physical education classes. Theory Res Educ 2009;7(2):194-202.

14. Taylor IM, Ntoumanis N. Teacher motivational strategies and student self-determination in physical education. J Educ Psychol 2007;99(4):747-760.

15. Chatzisarantis NLD, Hagger MS. Effects of an intervention based on self-determination theory on self-reported leisure-time physical activity participation. Psychol Health 2009;24(1):29-48.

16. Moreno Murcia JA, Parra Rojas N, González-Cutre Coll D. Influencia del apoyo a la autonomia, las metas sociales y la relacion con los demas sobre la desmotivacion en educacion fisica. Psicothema 2008;20:636641.

17. Standage M, Duda JL, Ntoumanis N. Students' motivational processes and their relationship to teacher ratings in school physical education: A self-determination theory approach. Res Q Exerc Sport 2006;77(1):100-110.

18. Vlachopoulos SP. The role of self-determination theory variables in predicting middle school students' subjective vitality in physical education. Hellenic J Psychol 2012;9(2):179-204.
19. Sánchez-Oliva D, Leo FM, Amado D, Cuevas R et al. Desarrollo y validación del cuestionario de apoyo a las necesidades psicológicas básicas en educación física. Mot Eur J Hum Mov 2013;30:53-71.

20. Van den Berghe L, Vansteenkiste M, Cardon G, Kirk D et al. Research on self-determination in physical education: key findings and proposals for future research. Phys Educ Sport Pedagog 2014;19(1):97-121.

21. Viira R, Koka A. Participation in afterschool sport: relationship to perceived need support, need satisfaction, and motivation in physical education. Kinesiology 2012;44(2):199-208.

22. Zhang T, Solmon MA, Gu XL. The role of teachers' support in predicting students' motivation and achievement outcomes in physical education. J Teach Phys Educ 2012;31(4):329-343.

23. Zamarripa J, Castillo I, Tomás I, López-Walle J. Validación Mexicana del Cuestionario de Apoyo a las Necesidades Psicológicas Básicas. Rev Mex Psicol (en prensa).

24. Goudas M, Biddle S, Fox K. Perceived locus of causality, goal orientations, and perceived competence in school physical education classes. Br J Educ Psychol 1994;64(3):453-463.

25. Ebbeck V, Weiss MR. Determinants of children's self-esteem: an examination of perceived competence and affect in sport. / Determinants de l'amour propre des enfants: etude de leur perception de leur propre competence et de leur affects en sport. Pediatr Exerc Sci 1998;10(3):285298.

26. Merino MD, Privado J, Gracia Z. Validación mexicana de la Escala de Funcionamiento Psicológico Positivo. Perspectivas en torno al estudio del bienestar y su medida. Salud Ment 2015;38(2):109-115.

27. Cheung, GW, Rensvold, RB. Evaluating Goodness-of-Fit Indices for testing measurement invariance. Struct Equ Modeling 2002;9:233-255.

28. Marsh HW, Hau KT, Wen Z. In search of golden rules: comment on hypothesis-testing approaches to setting cutoff values for fit indexes and dangers in overgeneralizing $\mathrm{Hu}$ and Bentler's (1999) findings. Struct Equ Modeling 2004;11:320-341.

29. Chen, FF. Sensitivity of goodness of $\mathrm{fi} t$ indexes to lack of measurement invariance. Struct Equ Modeling 2007;14:464-504.

30. Jöreskog K, Sörbom D. LISREL 8.80. Structural equation modeling with the simplis command language (Software de cómputo). Chicago, EU: Scientific Software International; 2006.

31. MacKinnon DP. Introduction to statistical mediation analysis. Mahwah, NJ: Erlbaum; 2008.

32. Taylor AB, MacKinnon DP, Tein J. Tests of the three-path mediated effect. Organ Res Methods 2008;11:241-269.

33. MacKinnon DP. Contrasts in multiple mediator models. En: Rose JS, Chassin L, Presson CC, Sherman SJ (eds.). Multivariate applications in substance use research. Mahwah, NJ: Lawrence Erlbaum Associates, Inc; 2000; pp.141-160. 